\title{
Stage III and IV Head and Neck Cancer: Does Everyone Need Chemotherapy?
}

\author{
Gaurav Marwaha, William L. Barrett \\ Department of Radiation Oncology, University of Cincinnati College of Medicine, Cincinnati, USA \\ Email: marwahg2@ccf.org, William.Barrett@UCHealth.com
}

Received August 21, 2013; revised September 20, 2013; accepted October 10, 2013

Copyright (C) 2014 Gaurav Marwaha, William L. Barrett. This is an open access article distributed under the Creative Commons Attribution License, which permits unrestricted use, distribution, and reproduction in any medium, provided the original work is properly cited. In accordance of the Creative Commons Attribution License all Copyrights (C 2014 are reserved for SCIRP and the owner of the intellectual property Gaurav Marwaha, William L. Barrett. All Copyright @ 2014 are guarded by law and by SCIRP as a guardian.

\begin{abstract}
Objectives: Definitive treatment of Stage III and IV squamous cell carcinoma can be with surgical resection, definitive radiation therapy alone or combined radiation therapy with chemotherapy. Radiation and concomitant platinum-based chemotherapy are the accepted gold standard. The purpose of this study was to determine how often patients treated with radiation therapy alone developed locoregionally recurrent disease that in retrospect possibly could have been prevented with the addition of chemotherapy. Methods: 116 consecutive patients with known Stage III and Stage IV head and neck cancers were treated with curative intent with radiation therapy alone. Results of the treatment were retrospectively reviewed. Results: Of the 116 patients treated with radiation alone, $11(9.48 \%)$ died from locally recurrent disease, 6 (5.17\%) died from local disease and were never diseasefree, 7 (6.03\%) died from metastatic disease, 9 (7.75\%) died from disease NOS, 6 (5.17\%) died from secondary malignancy, 10 (8.62\%) died from ICD (2 oropharynx; 8 larynx), 6 (5.17\%) died from uncertain causes, 51 $(43.96 \%)$ are alive and disease-free, and $10(8.62 \%)$ patients' final outcome data were not recoverable. Conclusions: Cure rates in selected patients with advanced head and neck cancer may be similar with radiation alone compared to radiation with the addition of chemotherapy.
\end{abstract}

\section{KEYWORDS}

\section{Head and Neck Cancer; Concurrent Chemoradiation; Definitive Radiation}

\section{Introduction}

In the United States, the annual incidence of head and neck squamous cell carcinomas (HNSCC) approached 36,000 for 2011 [1]. Worldwide, HNSCC incidence has reached half a million cases annually, making it the fifth most common cancer [2].

Definitive treatment of Stage III and IV squamous cell carcinoma can be with surgical resection (frequently followed by adjuvant radiation therapy or radiation with platinum-based chemotherapy), definitive radiation therapy alone or combined radiation therapy with chemotherapy. In this era, nationwide, these cancers are most commonly treated with combined radiation and chemotherapy with the expectation that the likelihood of disease eradication will be higher than that with radiation therapy alone, albeit with greater treatment-related toxicity.
In a review of 87 trials and 16,487 patients with locally advanced non-metastatic HNSCC, it was reported that an absolute survival benefit of $4.5 \%$ at 5 years exists with the addition of chemotherapy [3]. No clear benefit was associated with induction or adjuvant chemotherapies, but an absolute benefit of $6.5 \%$ at 5 years with concomitant chemotherapy was reported [3]. Radiation and concomitant platinum-based chemotherapy is the accepted gold standard based on the largest multicenter randomized trials [4].

Concurrent chemotherapy and radiation can come at a high cost, where acute toxicity is significant and can even cause death [5]. Furthermore, patients with head and neck cancer frequently have comorbidities that can impair their survival irrespective of their cancer. Treatment of elderly patients is particularly difficult in this 
light [6]. Additionally, patients who are cured of their primary head and neck cancer are at risk of second cancers, which may further impair their survival.

There are numerous potential causes of death in stage III and IV head and neck cancer, including intercurrent disease, second primary cancers, locoregionally recurrent disease, and metastatic disease [7-9].

Data comparing radiation therapy alone versus chemoradiation are limited to studies that generally cluster Stage III and IV patients together, and usually do not take into account prognostic factors that may predict outcomes for patients treated with radiation alone versus chemoradiation. In the authors' experience, chemotherapy has been reserved for only the more advanced cancers, so as to limit toxicity in radiation side effects in patients that are thought to have a reasonable chance for cure with radiation therapy alone.

Most failures of treatment of advanced HNSCC are due to locoregional recurrence. Thus, the purpose of this study was to determine how often patients treated with radiation therapy alone developed locoregionally recurrent disease that in retrospect possibly could have been prevented with the addition of chemotherapy.

\section{Materials and Methods}

Between 1995 and 2007, 116 consecutive patients with known Stage III (any T3 or T1-3N1 by AJCC: American Joint Committee on Cancer, $7^{\text {th }}$ edition) and Stage IV (any T4 or T1-4N2-3 by AJCC) head and neck cancers (30 with cancers of the larynx; 86 with cancers of the oropharynx) were treated with curative intent by one radiation oncologist with radiation therapy alone at the University of Cincinnati.

Radiation therapy alone consisted of conventional 3dimensional (3D) conformal external beam radiation therapy (EBRT) with median dose delivered to primary site of 70 Gray (Gy). Volumes treated reflected disease burden and the discretion of the treating physician. Median follow-up was 3.83 years for laryngeal cancer patients and 3.88 years for oropharyngeal cancer patients.

Results of the radiation treatments and patient outcomes were retrospectively reviewed with the approval of the Institutional Review Board (IRB). Patient clinical records, the University Hospital tumor registry, hospital records, and the Social Security Death Index (SSDI) were all utilized to construct the patient data set, which included cause of death.

Endpoints of investigation in this study included: survival, locoregional recurrence, distant metastasis, death rates from second primary cancers, and death rates from intercurrent disease. Cumulative incidences for these statistical endpoints were calculated for the cohort of patients in this study.

\section{Results}

Of the 116 total patients, 51 (43.96\%) were alive and disease-free (36 oropharynx; 15 larynx) at a median follow-up of 5 years (range: 3 months to 11 years), 34 (29.3\%) had achieved 5-year disease free survival (23 oropharynx; 11 larynx) and 70 (60.34\%) experienced a 2-year period of disease-free survival (48 oropharynx, 22 larynx).

Eleven of the 116 patients (9.48\%) died from locally recurrent disease ( 9 oropharynx; 2 larynx), 6 (5.17\%) died from disease and were never disease- free (5 oropharynx; 1 larynx), 7 (6.03\%) died from metastatic disease (6 oropharynx; 1 larynx), 9 (7.75\%) died from disease not otherwise specified (8 oropharynx; 1 larynx), 6 (5.17\%) died from secondary malignancy (4 oropharynx; 2 larynx), 10 (8.62\%) died from intercurrent disease (2 oropharynx; 8 larynx), 6 (5.17\%) died from uncertain causes (6 oropharynx), 51 (43.96\%) were rendered disease-free from their primary disease (36 oropharynx; 15 larynx) and 10 (8.62\%) patients' final outcome data were not recoverable (10 oropharynx). Figure 1 highlights the outcomes in each disease site group, respectively.

\section{Discussion}

Cure rates in selected patients with advanced head and neck cancer may be similar with radiation alone compared to those reported in the literature treated with radiation and chemotherapy. In this retrospective review, $14.65 \%$ of patients treated with radiation therapy alone died with locoregionally recurrent disease $(9.48 \%)$ or locoregional persistence of disease (5.17\%) and possibly

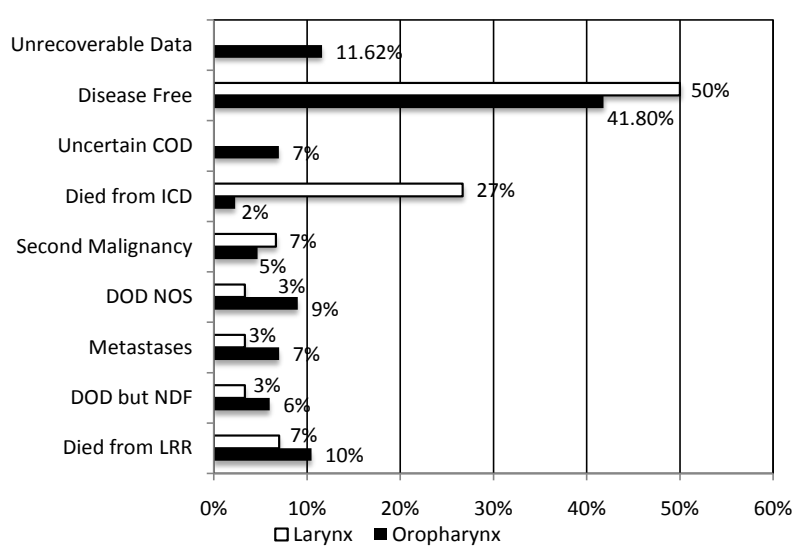

Figure 1. Outcomes for stage III-IV head and neck cancer patients receiving radiation alone. Shaded bars represent the percentage of oropharyngeal cancer patients with specific outcome and non-shaded bars represent laryngeal cancer patients. $(C O D=$ cause of death, $D O D=$ died of disease, ICD = intercurrent disease, NOS = not otherwise specified, $\mathrm{NDF}=$ never disease free, $\mathrm{LRR}=$ locoregional recurrence.) 
could have benefited from the addition of chemotherapy.

As some patients are rendered locoregionally diseasefree with radiation alone, the addition of chemotherapy in those patients adds only toxicity. Specifically, platinumbased chemotherapy can cause: gastrointestinal distress (nausea, vomiting, and diarrhea), infection secondary to neutropenia, anemia often requiring transfusions, ototoxicity, and nephrotoxicity. Additionally, side effects may be even more pronounced in patients with poorer performance status, and can even result in death. However, for otherwise medically fit patients who have less radiocurable disease (i.e., larger volume, particularly infiltrative characteristics), there is the benefit from the addition of chemotherapy, and it is those patients for whom we have reserved its use. These patients experience the powerful "radiosensitizing" effects from platinum-based chemotherapy, and in turn, an overall survival advantage.

The relatively low locoregional recurrence rate in the selected patients for radiation therapy alone validates the concept that clinical discretion has merit in treatment recommendations. The high rate of deaths from causes other than locoregional disease is consistent with other series and further mitigates any advantage to more aggressive, and more toxic, treatment.

One of the limitations of this study is the unknown HPV and/or p16 status in the oropharynx patients. These patients would likely have fared better overall due to the better prognosis associated with HPV-positive cancers and likely would have had disease more amenable to treatment with radiation alone.

One of the fundamental questions that needs to be answered is whether it is possible to systematically identify patients who require combined chemoradiation therapy and those who have a very high likelihood of cure with radiation therapy alone [4]. Meanwhile, this study highlights the importance of clinical discretion in treatment of advanced HNSCC where the patients' quality of life should be highly regarded and minimizing treatment toxicity is a priority.

Does everyone with Stage III or IV head and neck cancer treated with radiation therapy require concurrent chemotherapy? No. Many patients are cured with radiation therapy alone and those patients can be relatively reliably identified. In the current series, the radiocurable patients were relatively reliably (15\% locoregional persistence or recurrence rate) identified on clinical grounds and the development of biopredictors (i.e., HPV status) may further improve the accuracy of pretreatment determination of patients for whom chemotherapy can be avoided.

\section{REFERENCES}

[1] SEER*Stat Database: NAACCR Incidence-CiNA+. Surveillance, Epidemiology and End Results (SEER) Program. www.seer.cancer.gov.

[2] M. D. Parkin, F. Bray, J. Ferlay, et al., "Estimating the World Cancer Burden: Globocan 2000,” International Journal of Cancer, Vol. 94, No. 2, 2001, pp. 153-156. http://dx.doi.org/10.1002/ijc.1440

[3] J. P. Pignon, A. Maitre, E. Maillard, et al., "Meta-Analysis of Chemotherapy in Head and Neck Cancer (MACHNC): An Update on 93 Randomised Trials and 17,346 Patients,” Radiotherapy and Oncology, Vol. 92, No. 1, 2009, pp. 4-14. http://dx.doi.org/10.1016/j.radonc.2009.04.014

[4] D. J. Adelstein, "Concurrent Chemoradiotherapy in the Management of Squamous Cell Cancer of the Oropharynx: Current Standards and Future Directions," International Journal of Radiation Oncology, Biology, Physics, Vol. 69, No. 2, 2007, pp. S37-S39. http://dx.doi.org/10.1016/j.ijrobp.2007.04.086

[5] N. P. Nguyen, C. Frank, C. C. Moltz, et al., “Aspiration Rate Following Chemoradiation for Head and Neck Cancer, An Underreported Occurrence,” Radiotherapy and Oncology, Vol. 80, No. 3, 2006, pp. 302-306. http://dx.doi.org/10.1016/j.radonc.2006.07.031

[6] A. Sanabria, A. L. Carvalho, J. G. Vartanian, et al., “Comorbidity Is a Prognostic Factor in Elderly Patients with Head and Neck Cancer,” Annals of Surgical Oncology, Vol. 14, No. 4, 2007, pp. 1449-1457.

http://dx.doi.org/10.1245/s10434-006-9296-1

[7] E. E. Vokes, R. R. Weichselbaum, S. Lippman, et al., "Head and Neck Cancer," New England Journal of Medicine, Vol. 328, No. 3, 1993, pp. 184-194. http://dx.doi.org/10.1056/NEJM199301213280306

[8] S. M. Lippman and W. K. Hong, "Second Malignant Tumors in Head and Neck Squamous Cell Carcinoma: The Overshadowing Threat for Patients with Early-Stage Disease," International Journal of Radiation Oncology, Biology, Physics, Vol. 17, No. 3, 1989, pp. 691-694. http://dx.doi.org/10.1016/0360-3016(89)90126-0

[9] A. Argiris, B. E. Brockstein, D. J. Haraf, et al., “Competing Causes of Death and Second Primary Tumors in Patients with Locoregionally Advanced Head and Neck Cancer Treated with Chemoradiotherapy,” Clinical Cancer Research, Vol. 10, 2004, pp. 1956-1962. http://dx.doi.org/10.1158/1078-0432.CCR-03-1077 